Research efforts involving several disciplines: adherence to a clear nomenclature is needed

Philippe C. Baveye, John Palfreyman, Wilfred Otten

This is the authors' final version of this article. The final publication is available at Springer via http://dx.doi.org/10.1007/s11270-014-1997-7 
*Corresponding author. E-mail: baveye.rpi@gmail.com (6), art. no. 1997 .

\section{Environmental research efforts involving several disciplines: Adherence to a clear nomenclature is needed}

\author{
Author's accepted version
}

Please cite as

\section{Philippe C. Baveye ${ }^{1,2^{*}}$, John Palfreyman ${ }^{1}$, Wilfred Otten ${ }^{1}$ \\ ${ }^{1}$ SIMBIOS Centre, Abertay University, Kydd Building, 40 Bell Street, Dundee DD1 1HG, Scotland \\ ${ }^{2}$ Soil and Water Laboratory, Department of Civil and Environmental Engineering, Rensselaer Polytechnic Institute, 110 8th street, Troy, New York 12180, USA}


According to Google Scholar, more than 1.6 million scholarly articles and book chapters have been published to date, which in one way or another describe "interdisciplinary" research efforts. Many scientific periodicals, like Water, Air and Soil Pollution, include the term "interdisciplinary" in their full, official title, and their number is steadily growing. Likewise, more and more research institutes are devoted explicitly to interdisciplinary endeavors.

This attractiveness of venues where several disciplines meet to address pressing research questions is perhaps nowhere clearer than in relation to environmental concerns. In most areas in this field, the research community is now confronted with issues of such a complexity and technical difficulty that they preclude simple treatments carried out entirely within the confines of individual disciplines, like environmental physics, chemistry, or microbiology. To address most cases of environmental contamination or the numerous problems arising at a variety of spatial scales because of global climate change, input from different disciplinary perspectives, including those of economics and social sciences, is increasingly recognized as essential.

Many authors refer to research efforts involving several distinct disciplines as "interdisciplinary". Equally many adopt alternate terms, e.g., multi-, pluri-, cross-, or transdisciplinary, to describe what essentially appears to be the same thing. In some cases, the same terms are adopted to refer to very different concepts. For example, the term of "crossdisciplinarity" sometimes serves as an umbrella for multi-, inter-, and transdiciplinarity (e.g., Hinze, 1999; Wu, 2006), but occasionally is also used to designate research efforts 
that are very distinct in character from these "other"-disciplinarities. This profusion of terms has generated and continues to cause a great deal of confusion in practice, in particular when participants in research efforts involving distinct disciplines have very different perceptions of what their roles should be. Similarly, discrepancies between the expectations of funding agencies, research institutions, program managers, and individual researchers about the level of disciplinary integration that should be targeted in any given "interdiscisplinary" effort can lead to utter confusion, and may explain to some extent why so many of theses efforts are reported to stumble or even fail altogether (e.g., Hicks et al., 2010). Based on our experience, we feel that these issues can be resolved in part by agreeing on a carefully thought-out set of definitions.

To a large extent, confusion among the different concepts of multi-, inter-, cross-, and trans-disciplinarity could have been alleviated quite a few years ago if more attention had been paid to the very insightful paper of Tress et al. (2004). These authors not only defined clearly a number of these terms, but also proposed a clever and extremely insightful depiction of the relationships among disciplines that the terms embody. Their work constitutes an ideal starting point to propose a new, updated nomenclature (Figure 1), as will be done in the following by introducing into it the additional term of "crossdisciplinarity", in its narrow acception, and by expanding slightly the notion of interdisciplinarity itself into two successive stages.

The description of the expanded nomenclature needs to start with the concept of disciplinarity, which is fundamental to any classification in this area (Tress et al., 2004). A discipline has its own coherent set of tools, methods, procedures, concepts and theories. Disciplines are shaped by external conditions and internal intellectual demands. As a result, 
their boundaries tend to be somewhat arbitrary and shifting over time. Disciplines organize experiences into a certain worldview. They work within a specific framework of beliefs and criteria for truth and validity that limits the kind of research questions that can be asked. As illustrated in Fig. 1, in a strictly disciplinary approach, no systematic relations or conceptual exchanges occur among disciplines. Each discipline sets its own goals, and in the process of reaching them, produces new disciplinary knowledge and theories.

Strict disciplinarity is an extreme situation. In many cases, researchers working within the boundaries of one discipline often make use of concepts and techniques developed in other disciplines. A prototypical example of this is the fact that Einstein's work on relativity could not have seen the light of day if he had not been introduced to a then-esoteric theory (tensor calculus) developed by mathematicians for entirely different purposes. Similarly, countless measurement and visualization techniques, as well as statistical and numerical methods, have been adopted by researchers in many disciplines in order to enable them to carry out their work. In the literature (e.g., Butzer, 2005; Wodak, 2007), this type of crossfertilization among disciplines, where aspects of one discipline are explained in terms of one or more other disciplines, has been referred to as cross-disciplinarity (Figure 1). It shares with strict disciplinarity the feature that research objectives are set within the confines of each discipline, and eventually contribute new knowledge only in that context.

The term of multidisciplinarity refers to "research efforts of different academic disciplines that relate to a shared goal, but with multiple disciplinary objectives. Participants exchange knowledge, but they do not aim to cross subject boundaries in order to create new integrative knowledge and theory" (Tress et al., 2004). For example, environmental scientists might get together with economists to determine, for a particular 
geographical area, which one of a number of renewable energy supplies makes the most technical and economical sense. Within such a project, each discipline would have its own disciplinary objective. Neither discipline will be particularly affected in the long term by the interaction, and the collaboration could well finish once the research question has been answered. The benefit of the multidisciplinary approach is that each discipline adds new knowledge from its own perspective to complete the picture like pieces in a jigsaw puzzle (e.g., Thevenon and Pote, 2012).

Cross-disciplinarity and multidisciplinarity are characterized by low integration of two or more disciplines, which tend to work pretty much in parallel. On the contrary, interdisciplinarity and transdisciplinarity, occasionally lumped together under the umbrella term of integrative research (Fig. 1), correspond to a significantly higher level of integration among disciplines.

NAS (2004) defines interdisciplinarity as "a mode of research by teams or individuals that integrates information, data, techniques, tools, perspectives, concepts and/ or theories from two or more disciplines or bodies of specialised knowledge to advance fundamental understanding or to solve problems whose solutions are beyond the scope of a single discipline area or area of research practice." In other words, like multidisciplinarity, interdisciplinarity involves several unrelated academic disciplines, each with their own contrasting research paradigms, but it does so in a way that forces them to cross subject boundaries. In the process of striving toward a common research goal, the concerned disciplines integrate disciplinary knowledge in order to create new knowledge and theory. After it has emerged, this new insight can no longer be broken down into its disciplinary ingredients. The necessary breaking down of disciplinary barriers in more and more cases 
is so severe that some authors have argued that the term of antidisciplinarity (Mowitt, 1992; Rosow, 2003) is more appropriate. It may yet become the preferred terminology, especially after being espoused recently by MIT's Media Lab, but for the time being, interdisciplinarity is more widely accepted.

Tress et al. (2004) point out that one of the greatest challenges of integrative research is to bring together different epistemologies, which requires "researchers to become immersed in one another's knowledge cultures, to understand the fundamental differences in their basic theories and axioms." The need for participants in interdisciplinary research efforts to have "intellectual objectives, epistemological convictions, and professional strategies [that] are orthogonal to many of the disciplinary-based practices of the academy" (Rhoten and Pfirman, 2007), often leads to frictions with traditional institutional structures, which need to be alleviated. A second challenge is related to the fact that, in order to identify a common, interdisciplinary objective for a project, participating disciplines must focus their efforts towards integration from the very beginning of the research endeavour. This requires participants to have a clear idea of why an interdisciplinary approach is required for the problem under investigation. A full agreement needs to exist on how interdisciplinarity should be understood and implemented in the project, which can be achieved by setting goals that are clearly linked to interdisciplinary efforts. When this does not happen, it is very easy for research efforts, despite their intention to be interdisciplinary, to drift toward multidisciplinarity (e.g., Rhoten, 2004; Rhoten and Parker, 2004; Baveye, 2013a,b).

Compared to the diagram in Tress et al. (2004), Figure 1 introduces two successive stages under interdisciplinarity. Initially, as individuals from distinct disciplines interact 
across disciplinary boundaries to make progress toward their common goal, there is no particular structure to this interaction. However, over time, as the interaction evolves and intensifies, new methods and perspectives are developed, which when they have reached a sufficient level of maturity, can form the foundation of a new, emerging discipline, especially if a number of research projects, involving a critical mass of participants, are moving in the same direction. In many ways, one could argue that in the past, because of the scope of the problems tackled, interdisciplinary efforts tended to remain at the first stage, whereas nowadays, they frequently move on to the second stage. Certainly, the meteoric rise of systems biology (Noble, 2006), or the rapid development of quantum microbiology (Trevors and Masson, 2011) over the last few years, signal a much quicker transition to the creation of new disciplines than was the case in earlier times.

The last of the terms depicted in Figure 1 is that of transdisciplinarity. Transdisciplinary research, a relatively new idea, corresponds to projects that involve academics from different unrelated disciplines as well as non-academic participants, belonging to various categories of stakeholders, to jointly create new knowledge and theory as they try to address a common question (Tress et al., 2004). In other words, transdiciplinarity transcends academia, deals with relevant, complex societal problems, and organizes processes of mutual learning between agents from the scientific and the non-scientific words (Scholz et al., 2000), i.e., between researchers and societal or industrial participants. In this sense, it represents the ultimate form of integrative research.

Now that this new, improved nomenclature is available, the question is how to make the best use of it. One possibility would be to request that, in proposals submitted for funding, researchers use this nomenclature to describe accurately the nature and the modus 
operandi of the work that they anticipate doing. Funding bodies that explicitly demand interdisciplinary, or even transdisciplinary research, would probably benefit from using the scheme depicted in Figure 1 to explain clearly what they mean by these terms. However, merely demanding that people submitting research proposal refer to interdisciplinarity in their texts does not guarantee at all that the research carried out eventually be interdisciplinary in more than intent. Funding bodies should also request clear evidence of the existence of research management structures that foster integrative collaboration.

Other possible uses of the nomenclature of Figure 1 may be more after the fact, at the publication stage. One option would be for editors and associate editors of journals like Water, Air and Soil Pollution to ask authors of manuscripts to make explicit use of the nomenclature to describe faithfully the nature of past research efforts they are reporting on in their writing. Reviewers could also, systematically, be asked to check that the nomenclature is used appropriately in the manuscripts they are assigned. A different route to the same end would consist of asking authors to provide detailed information about the way the research described in their article was carried out, in terms for example of who came up with the ideas, how the workload was divvied up, who put the manuscript together. For an effort to be truly interdisciplinary, one would expect that most aspects of the work would be shared equally among the participants from the different disciplines. If authors were encouraged through these various mechanisms to give a consistent and realistic account of the type of collaborative research effort they engaged in and which eventually led to their articles, not only would this inform readers better on what they can expect to gain from reading the articles in question, but also it would provide the scientific 
community in general a more reliable perception of the importance of truly interdisciplinary efforts, relative to the mass of research projects carried out every year. It would be an eye opener in this respect if, amidst the overwhelming claims that interdisciplinary research is absolutely crucial to address numerous societal concerns, in terms of the environment or in other respects, we found out that, in reality, very few research projects actually manage to be interdisciplinary and many, as a result, do not deliver their projected outcomes.

\section{References}

Baveye, P.C. (2013a). Comment on "Ecological engineers ahead of their time: The functioning of pre-Columbian raised-field agriculture and its potential contributions to sustainability today" by Dephine Renard et al., Ecological Engineering, 52(1), 224-227.

Baveye, P.C. (2013b). Addressing key challenges to interdisciplinary research on water-related issues: Biologists' engagement and funding structure. Biologia, 68(6), 1087-1088.

Butzer, K.W. (2005). Environmental history in the Mediterranean world: Cross-disciplinary investigation of cause-and-effect for degradation and soil erosion. Journal of Archaeological Science, 32, 1773-1800.

Hicks, C.C., Fitzsimmons, C., \& Polunin, N.V.C. (2010). Interdisciplinarity in the environmental sciences: barriers and frontiers. Environmental Conservation, 37, 464-477.

Hinze, S. (1999). Collaboration and cross-disciplinarity in autoimmune diseases. Scientometrics, 46, 457-471.

Mowitt, J. (1992). Text: The Genealogy of an Antidisciplinary Object. Duke University Press, Durham, North Carolina.

National Academy of Sciences (2004). Facilitating interdisciplinary research. National Academies Press, Washington, D.C. 
Noble, D. (2006). The Music of Life: Biology Beyond Genes. Oxford University Press, Oxford, UK.

Rhoten, D. (2004). Interdisciplinary research: Trend or transition. Items \& Issues, Spring/Summer, 6-11.

Rhoten, D., \& Parker, A. (2004). Risks and rewards of an interdisciplinary research path. Science, $306,2046-2046$.

Rhoten, D., \& Pfirman, S. (2007). Women in interdisciplinary science: Exploring preferences and consequences. Research Policy, 36, 56-75.

Rosow, S.J. (2003). Toward an anti-disciplinary global studies. International Studies Perspectives. 4, $1-14$.

Scholz, R.W., Mieg, H.A., \& Oswald, J.E. 2000. Transdisciplinarity in groundwater management Towards mutual learning of science and society. Water Air and Soil Pollution, 123(1-4), 477487.

Thevenon, F., \& Pote, J. (2012). Water pollution history of Switzerland recorded by sediments of the large and deep perialpine lakes Lucerne and Geneva. Water Air and Soil Pollution, 223(9), 6157-6169.

Tress, G., Tress, B., \& Fry, G. (2005). Clarifying integrative research concepts in landscape ecology. Landscape Ecology. 20, 479-493.

Trevors, J.T., \& Masson, L. (2011). Quantum microbiology. Current Issues in Molecular Biology, 13(2), 43-49.

Wodak, R. (2007). Pragmatics and critical discourse analysis. A cross-disciplinary inquiry. Pragmatics and Cognition, 15, 203-225.

Woelert, P., \& Millar, V. (2013). The 'paradox of interdisciplinarity' in Australian research governance. Higher Education, 66, 755-767.

Wu, J.G. (2006). Landscape ecology, cross-disciplinarity, and sustainability science. Landscape Ecology, 21, 1-4. 


\section{Level of integration}

\section{Parallel}

\section{Integrative}



